\title{
Correction to: Anonymous shell companies: A global audit study and field experiment in 176 countries
}

Brent B Allred ${ }^{1}$,

Michael G Findley², Daniel Nielson ${ }^{3}$ and J C Sharman ${ }^{4}$

\footnotetext{
${ }^{1}$ Raymond A. Mason School of Business, The College of William \& Mary, Williamsburg, VA, USA; ${ }^{2}$ Department of Government, University of Texas at Austin, Austin, TX, USA; ${ }^{3}$ Department of Political Science, Brigham Young University, Provo, UT, USA; ${ }^{4}$ Department of Politics and International Studies, University of Cambridge, Cambridge, UK
}

Correspondence:

BB Allred, Raymond A. Mason School of Business, The College of William \& Mary, Williamsburg, VA, USA

e-mail: allred@mason.wm.edu
Journal of International Business Studies (2018) 49, 246-247.

https://doi.org/ | 0.1057/s4 | 267-017-0135-3

\section{CORRECTION TO: JOURNAL OF INTERNATIONAL BUSINESS STUDIES (2017) 48, 596-619 https://doi.org/10.1057/s41267-016-0047-7}

Owing to a production error, a number of values were omitted in Table 1 of the original article. A corrected version of this table is provided here. The corrected values are italicized. 
Table 1 Displays difference in means tests for the total sample, OECD countries, Tax Haven countries, and Developing countries (row blocks) for the five possible outcomes of no-reply, non-compliant, part-compliant, compliant, \& refusal (rows within blocks) for the Placebo, FATF, Consequences, and Appropriateness experimental conditions (columns)

Response Placebo FAFT (information on law) $\quad$ Sig. Rationalism (penalties) Sig. Constructivism (norms) Sig.

Comparison of means between placebo and treatments - total sample

$\begin{array}{lrr}\text { No reply } & 44.75 \% & 49.46 \% \\ \text { Non-compliant } & 8.42 \% & 8.11 \% \\ \text { Partial compliant } & 16.65 \% & 16.22 \% \\ \text { Compliant } & 19.49 \% & 16.76 \% \\ \text { Refusal } & 10.69 \% & 9.46 \% \\ \text { Total } & 100.00 \% & 100.00 \% \\ \mathrm{n} & 1057 & 370\end{array}$

Comparison of means between placebo and treatments - OECD countries

$\begin{array}{lrrr}\text { No reply } & 44.72 \% & 46.81 \% & 45.06 \% \\ \text { Non-compliant } & 13.73 \% & 14.89 \% & 10.99 \% \\ \text { Partial compliant } & 15.14 \% & 13.83 \% & 15.39 \% \\ \text { Compliant } & 14.09 \% & 8.51 \% & 18.68 \% \\ \text { Refusal } & 12.32 \% & 15.96 \% & 9.89 \% \\ \text { Total } & 100.00 \% & 100.00 \% & 100.00 \% \\ \mathrm{n} & 284 & 94 & 91\end{array}$

$\begin{array}{rr}48.63 \% & 50.00 \% \\ 7.38 \% & 9.78 \% \\ 19.95 \% & 14.13 \% \\ 16.39 \% & 16.03 \% \\ 7.65 \% & 10.05 \% \\ 100.00 \% & 100.00 \% \\ 366 & 368\end{array}$

Comparison of means between placebo and treatments - Tax Haven countries

\begin{tabular}{|c|c|c|c|c|c|c|}
\hline No reply & $34.78 \%$ & $25.74 \%$ & + & $45.28 \%$ & + & $22.89 \%$ \\
\hline Non-compliant & $4.01 \%$ & $4.95 \%$ & & $3.77 \%$ & & $8.43 \%$ \\
\hline Partial compliant & $15.72 \%$ & $24.75 \%$ & * & $22.64 \%$ & & $18.07 \%$ \\
\hline Compliant & $39.80 \%$ & $40.59 \%$ & & $23.59 \%$ & $* *$ & $36.15 \%$ \\
\hline Refusal & $5.69 \%$ & $3.96 \%$ & & $4.72 \%$ & & $14.46 \%$ \\
\hline Total & $100.00 \%$ & $100.00 \%$ & & $100.00 \%$ & & $100.00 \%$ \\
\hline $\mathrm{n}$ & 299 & 101 & & 106 & & 83 \\
\hline
\end{tabular}

Comparison of means between placebo and treatments - developing countries

\begin{tabular}{|c|c|c|c|c|c|c|}
\hline No reply & $51.06 \%$ & $64.57 \%$ & ** & $52.66 \%$ & $61.49 \%$ & * \\
\hline Non-compliant & $8.02 \%$ & $6.29 \%$ & & $7.69 \%$ & $9.20 \%$ & \\
\hline Partial compliant & $18.14 \%$ & $12.57 \%$ & + & $20.71 \%$ & $13.79 \%$ & \\
\hline Compliant & $9.92 \%$ & $7.43 \%$ & & $10.65 \%$ & $9.77 \%$ & \\
\hline Refusal & $12.87 \%$ & $9.14 \%$ & & $8.28 \%$ & $5.75 \%$ & * \\
\hline Total & $100.00 \%$ & $100.00 \%$ & & $100.00 \%$ & $100.00 \%$ & \\
\hline$n$ & 474 & 175 & & 169 & 174 & \\
\hline
\end{tabular}

Each entry refers to the percent of subjects in a country block who received a given condition and responded in a certain way. Those percentages are accompanied by significance tests, which compare the FATF, Consequences, and Appropriateness conditions to the Placebo and identify when they are meaningfully different outcomes.

Sig. significance of difference between mean of Placebo and each treatment.

$+\mathrm{p}<0.10,{ }^{*} \mathrm{p}<0.05 ;{ }^{* *} \mathrm{p}<0.01 ;{ }^{* * *} \mathrm{p}<0.001$ 